\title{
Diagnóstico diferencial de dengue y chikungunya en pacientes pediátricos
}

\author{
Eliana Patricia Calvo ${ }^{1}$, Carolina Coronel-Ruiz', Syrley Velazco², \\ Myriam Velandia-Romero ${ }^{1}$, Jaime E. Castellanos ${ }^{1}$ \\ 1 Laboratorio de Virología, Universidad El Bosque, Bogotá, D.C., Colombia \\ 2 Hospital Universitario de La Samaritana, Unidad Funcional Girardot, Bogotá, D.C., Colombia
}

Introducción. Las infecciones por el virus del dengue y del chikungunya presentan síntomas clínicos similares, lo cual dificulta el diagnóstico clínico. Además, son transmitidas por los mismos vectores, por lo que en una región puede haber circulación e infección simultánea con los dos virus. Los resultados de cada enfermedad, no obstante, son diferentes: la fiebre del chikungunya rara vez es fatal, pero puede dejar secuelas de tipo articular y neurológico, en tanto que el dengue es potencialmente fatal. De ahí la importancia de un diagnóstico preciso y oportuno.

Objetivo. Comparar el diagnóstico presuntivo basado en los hallazgos clínicos con el diagnóstico diferencial hecho mediante pruebas de laboratorio.

Materiales y métodos. Se utilizaron pruebas virológicas y serológicas específicas para dengue y chikungunya en ocho muestras de sangre de pacientes pediátricos con síndrome febril. Se empleó la reacción en cadena de la polimerasa con transcriptasa inversa para detectar los virus del dengue y del chikungunya y el método de ELISA basado en la captura de lgM para confirmar los casos de dengue. Resultados. Con base en los hallazgos clínicos, dos pacientes se clasificaron como casos probables de dengue o chikungunya, dos como casos probables de chikungunya y en cuatro no hubo diagnóstico presuntivo de infección viral. Las pruebas de laboratorio confirmaron la infección por el virus del dengue en dos pacientes, por el virus del chikungunya en otros dos e infección simultánea de dengue y chikungunya en los cuatro restantes.

Conclusión. Los hallazgos clínicos no fueron suficientes para hacer un diagnóstico en pacientes pediátricos con síndrome febril, por lo cual se requirieron pruebas específicas de laboratorio para establecer con precisión el agente etiológico causante de la enfermedad.

Palabras clave: arbovirus, dengue, virus del chikungunya, coinfección, fiebre, diagnóstico, recién nacido. doi: http://dx.doi.org/10.7705/biomedica.v36i0.2982

\section{Dengue and Chikungunya differential diagnosis in pediatric patients}

Introduction: Dengue and Chikungunya infections have similar clinical symptoms, which makes their clinical diagnosis complex. Moreover, both are transmitted by the same mosquito vectors, which results in virus co-circulation and co-infection. However, the outcome of these diseases differs: Chikungunya fever is rarely fatal but can have permanent and severe rheumatic and neurological sequelae, whereas dengue disease is potentially fatal. Thus, accurate diagnosis is critical.

Objective: To compare presumptive diagnoses based on clinical findings with the differential diagnoses based on specific laboratory tests for each virus.

Materials and methods: We performed specific virological and serological tests for both dengue and Chikungunya infections on eight acute-phase blood samples collected from pediatric patients with febrile syndrome. We used RT-PCR to detect dengue and Chikungunya virus, and IgM-capture ELISA to confirm infection by dengue virus.

Results: Based on clinical findings, two patients were diagnosed as probable cases of dengue or Chikungunya, and two were diagnosed as probable cases of chikungunya. Four had no presumptive diagnosis of viral infection. Laboratory tests confirmed dengue infection in two patients, Chikungunya infection in two patients, and co-infection by the two viruses in the other four patients.

\footnotetext{
Contribución de los autores:

Eliana Patricia Calvo y Jaime E. Castellanos: concepción del estudio, análisis e interpretación de los datos

Eliana Patricia Calvo y Carolina Coronel-Ruiz: ensayos de laboratorio

Carolina Coronel-Ruiz y Myriam Velandia-Romero: análisis e interpretación de los datos

Syrley Velazco: revisión de historias clínicas

Eliana Patricia Calvo: escritura del documento

Todos los autores leyeron, revisaron y aprobaron el documento.
} 
Conclusion: Clinical findings were not sufficient to make a diagnosis in pediatric patients with febrile syndrome; specific laboratory tests were required to establish the etiologic agent of the disease.

Key words: Arboviruses, dengue, Chikungunya virus, coinfection, fever, diagnosis, infant, newborn. doi: http://dx.doi.org/10.7705/biomedica.v36i0.2982

Las enfermedades causadas por los virus transmitidos por artrópodos hematófagos, o arbovirus, constituyen uno de los principales problemas de salud pública en todo el mundo. Los arbovirus se encuentran principalmente en las regiones tropicales y subtropicales, y en la mayoría de los casos causan infecciones subclínicas, también denominadas fiebres inespecíficas $(1,2)$.

El virus del chikungunya (CHIKV) y el virus del dengue (DENV) son los arbovirus que tienen mayor impacto en la salud en las Américas, especialmente en Colombia (3-7). El CHIKV pertenece al género Alfavirus, familia Togaviridae, mientras que el DENV pertenece al género Flavivirus, familia Flaviviridae. Los dos tienen un genoma de ARN de cadena sencilla de polaridad positiva y ambos son transmitidos por los mismos vectores, los mosquitos Aedes aegypti y Aedes albopictus (2).

Los síntomas típicos causados por estos dos arbovirus incluyen fiebre, erupciones cutáneas, dolor muscular y articular, náuseas, dolor de cabeza y vómito, razón por la cual en algunas ocasiones las infecciones son clínicamente indistinguibles. Sin embargo, el resultado de cada enfermedad es diferente: la fiebre del chikungunya rara vez es fatal, pero puede dejar importantes secuelas de tipo articular y neurológico (8), en tanto que el dengue es potencialmente fatal (7). La infección con cualquiera de los cuatro serotipos del DENV produce un amplio espectro de manifestaciones clínicas que incluyen los cuadros clínicos con síntomas leves (denominados fiebre de dengue sin signos de alarma), otros con signos más evidentes (dengue con signos de alarma) y cuadros clínicos más graves (dengue grave), los cuales pueden tener un desenlace fatal (9). La infección con uno de los cuatro serotipos de DENV provoca una reacción inmunitaria que otorga protección de por vida contra nuevas infecciones con el mismo

\footnotetext{
Correspondencia:

Eliana Patricia Calvo, Laboratorio de Virología, Universidad El Bosque, Avenida Carrera 9 № 131A-02, edificio D, Bogotá, D.C., Colombia

Teléfono: (571) 648 9000, extensión 1209; Fax: (571) 6489066 calvoeliana@unbosque.edu.co

Recibido: 23/07/15; aceptado: 22/10/15
}

serotipo, pero puede potenciar la infección con otros serotipos y aumentar la probabilidad de desarrollar dengue con signos de alarma y dengue grave durante infecciones secundarias.

En las Américas, la circulación del DENV data de la década de los 80 ; desde entonces se ha extendido a cerca de 40 países de la zona (10). En Colombia, el número de casos ha crecido de manera importante en las últimas décadas y se caracteriza por brotes epidémicos cada tres 0 cuatro años $(7,11)$. En el 2010 , se presentó la epidemia más grande de los últimos años: se notificaron 157.152 casos, de los cuales 9.482 (6 \%) correspondieron a dengue grave (11). Aunque en los años siguientes el número de casos disminuyó hasta menos de 60.000, en 2013 y 2014 volvió a superar los 100.000 y hasta la semana 38 de 2015 se habían reportado 67.384 casos $(3,12)$.

En cuanto al CHIKV, el primer brote epidémico de la enfermedad en las Américas se reportó a finales del 2013 en la isla Saint Martin; desde entonces, la transmisión en la región ha ido en aumento y se ha extendido a 44 países y territorios. En estos dos años se han reportado cerca de 1,2 millones de casos sospechosos y 225 muertes (13). Hasta la semana epidemiológica 38 de 2015, se habían reportado 568.614 casos sospechosos, de los cuales 22.920 se confirmaron por laboratorio y 62 terminaron en muerte. En Colombia se han reportado 333.173 casos, de los cuales solo 1.961 se confirmaron por laboratorio y 55 fueron fatales (13). Cabe destacar que, desde la introducción del virus a mediados de 2014 hasta el momento, Colombia es el país con el mayor número de casos y de muertes reportadas (88 \%) en las Américas $(4,6,13)$.

Dado que los síntomas de la infección por el CHIKV son similares a los causados por el DENV, es posible que en las zonas donde el dengue es endémico el número de casos y de infecciones simultáneas se haya subestimado, o que los casos hayan pasado inadvertidos.

El diagnóstico clínico de estas arbovirosis en los primeros días de evolución es complejo, ya que presentan un cuadro febril agudo con síntomas 
inespecíficos que pueden confundirse con otras infecciones, como influenza, rubéola, gastroenteritis, fiebre tifoidea, fiebre amarilla y leptospirosis. Aunque existen varias pruebas virológicas (aislamiento viral, detección de antígeno NS1 o detección del ARN) y serológicas (presencia de lgM o lgG específicos) para la confirmación del diagnóstico de la infección por el DENV, en Colombia se utilizan principalmente en la investigación y en la vigilancia (14). En el caso del CHIKV, el diagnóstico se hace con base en el conjunto de manifestaciones clínicas, en tanto que la detección de ARN viral por reacción en cadena de la polimerasa (PCR) con transcriptasa inversa y la detección de IgM se usan principalmente para la investigación y la vigilancia $(3,4,6)$.

En el presente trabajo se evaluaron pacientes pediátricos con síndrome febril para comparar el diagnóstico presuntivo basado en los hallazgos clínicos con el diagnóstico diferencial basado en pruebas específicas de laboratorio. Se evidenció que el uso de los hallazgos clínicos y paraclínicos no fue suficiente para establecer un diagnóstico preciso y que las pruebas específicas de laboratorio para determinar el agente etiológico causante de la enfermedad son de gran importancia.

\section{Materiales y métodos}

\section{Muestras}

Se recolectaron de 3 a $5 \mathrm{ml}$ de sangre periférica de ocho pacientes pediátricos ingresados en febrero de 2015 al Hospital Universitario de La SamaritanaUnidad Funcional Girardot por síndrome febril no especificado.

La presente investigación se adelantó en el marco del proyecto "Establecimiento de un algoritmo de laboratorio para la confirmación de casos de dengue en muestras de individuos con síndrome febril atendidos en instituciones de salud de Cundinamarca", el cual cuenta con el aval del Comité de Ética del Centro de Investigación del Hospital y se viene desarrollando en Girardot, una población ubicada en Cundinamarca, a 289 msnm, con temperatura promedio de $29^{\circ} \mathrm{C}$ y humedad de $46 \%$.

Los criterios de inclusión fueron los siguientes: pacientes con síndrome febril de origen incierto de siete días de evolución o menos, cuyos signos y síntomas incluyeran fiebre alta, cefalea, dolor retroocular, mialgias, artralgias, postración, hemorragias, edema, exantema, hepatomegalia, esplenomegalia o alteración del estado neurológico.

\section{Revisión de historias clínicas}

Se revisaron las historias clínicas de los individuos y la información contenida en ellas se clasificó como hallazgos clínicos, exámenes de laboratorio clínico (hemograma, tiempo de protrombina, tiempo de tromboplastina parcial, entre otros) y diagnóstico presuntivo.

\section{Pruebas serológicas}

La detección de IgM para el DENV se hizo mediante ELISA con la prueba comercial UMELISA DENGUE IgM PLUS-Panbio ${ }^{\circledR}$, siguiendo el protocolo recomendado por el fabricante.

\section{Pruebas virológicas}

La detección de ARN viral se hizo mediante PCR semianidada. Se extrajo ARN a partir de $140 \mu \mathrm{l}$ de plasma, utilizando el estuche comercial QIAamp Viral RNA Mini Kit (Qiagen) y el protocolo recomendado por el fabricante. La detección de los dos virus se llevó a cabo mediante PCR semianidada $(15,16)$, según el protocolo modificado en el Laboratorio de Virología de la siguiente manera: a partir de $5 \mu \mathrm{l}$ de ARN y 0,2 $\mu \mathrm{M}$ de oligonucleótidos específicos para cada virus, se llevó a cabo la reacción de transcripción inversa y la amplificación con el sistema SuperScript III One-Step RT-PCR System with Platinum $\mathrm{Taq}^{\circledR}$ (Invitrogen). Luego se tomó $1 \mu \mathrm{l}$ del producto de la PCR como plantilla para una segunda reacción de amplificación con el sistema GoTaq ${ }^{\circledR}$ (Promega). La secuencia de los oligonucleótidos y el programa de amplificación utilizado se especifican en el cuadro 1.

\section{Definición de caso}

Se consideró como caso positivo de dengue, el paciente en quien se detectó el virus mediante PCR con transcriptasa inversa o anticuerpos IgM en plasma. El caso positivo de chikungunya se definió como el paciente en quien se detectó el virus mediante PCR con transcriptasa inversa.

\section{Resultados}

En el cuadro 2 se presentan los hallazgos clínicos y de laboratorio, así como el diagnóstico en el momento de ingreso de ocho pacientes pediátricos hospitalizados en el Hospital Universitario de La Samaritana, y los resultados de las pruebas de laboratorio específicas para el CHIKV y el DENV.

El grupo de pacientes estaba conformado por cuatro neonatos, dos menores de un año de edad y dos de cuatro y diez años de edad, los cuales 
Cuadro 1. Oligonucleótidos y programas de amplificación utilizados

\begin{tabular}{|c|c|c|}
\hline RT-PCR & Oligonucleótidos $5^{\prime}---3^{\prime}$ & Programa de amplificación \\
\hline DENV & $\begin{array}{l}\text { D1 TCAATATGCTGAAACGCGAGAGAAACCG } \\
\text { D2 TTGCACCAACAGTCAATGTCTTCAGGTTC } \\
\text { F ACGCAATTGAGCGAAGCAC }\end{array}$ & \multirow{2}{*}{$\begin{array}{l}\text { Transcripción inversa: a } 50^{\circ} \mathrm{C} \text { durante } 30 \text { minutos } \\
\text { Desnaturalización inicial: a } 95^{\circ} \mathrm{C} \text { durante } 3 \text { minutos } \\
\text { Desnaturalización: a } 95^{\circ} \mathrm{C} \text { durante } 30 \text { segundos } \\
\text { Hibridación: } 30 \text { ciclos a } 55^{\circ} \mathrm{C} \text { durante } 30 \text { segundos } \\
\text { Extensión: a } 72{ }^{\circ} \mathrm{C} \text { durante } 30 \text { segundos }\end{array}$} \\
\hline CHIKV & R CCAAATTGTCCYGGTCTTCCT & \\
\hline PCR & Oligonucleótidos5'----3' & Programa de amplificación \\
\hline CHIKV & $\begin{array}{l}\text { D1 TCAATATGCTGAAACGCGAGAGAAACCG } \\
\text { Ts1 CCCGTAACACTTTGATCGCT } \\
\text { Ts2 CGCCACAAGGGCCATGAACAGTTT } \\
\text { Ts3 TAACATCATCATGAGACAGAGC } \\
\text { Ts } 4 \text { TTCTCCCGTTCAGGATGTTC } \\
\text { F ACGCAATTGAGCGAAGCAC } \\
\text { R2 CTGAAGACATTGGCCCCAC }\end{array}$ & $\begin{array}{l}\text { Desnaturalización inicial: a } 95^{\circ} \mathrm{C} \text { durante } 3 \text { minutos } \\
\text { Desnaturalización: a } 95^{\circ} \mathrm{C} \text { durante } 30 \text { segundos } \\
\text { Hibridación: } 25 \text { ciclos a } 55^{\circ} \mathrm{C} \text { durante } 30 \text { segundos } \\
\text { Extensión: a } 72^{\circ} \mathrm{C} \text { durante } 30 \text { segundos }\end{array}$ \\
\hline
\end{tabular}

DENV: virus del dengue; CHIKV: virus del chikungunya; RT-PCR: reacción en cadena de la polimerasa con transcriptasa inversa

presentaron, además de fiebre, por lo menos un signo o síntoma típico de la infección con alguno de estos dos arbovirus. Cuatro de los pacientes fueron diagnosticados en el momento del ingreso como casos sospechosos de chikungunya por presentar erupción cutánea, debilidad e 'hipoactividad' y antecedentes familiares de infección (pacientes identificados con los códigos 371, 267, 958-3 y 986-1). En cuatro de los pacientes no se registró ninguna infección viral como diagnóstico presuntivo, aunque uno de ellos tenía síntomas y antecedentes familiares que sugerían la presencia del CHIKV (caso 511). Dos pacientes fueron diagnosticados como casos sospechosos de dengue (casos 371 y 267) y, aunque en otro paciente los hallazgos clínicos estaban asociados a dengue grave, el caso no se registró como tal (caso 420).

En cuanto a los exámenes de laboratorio, la prueba molecular permitió confirmar la infección por el CHIKV en seis de los pacientes (casos 371, $511,267,519,963$ y 986-1) y, con el DENV, en dos (casos 511, 420), en tanto que la detección de anticuerpos IgM para el DENV fue positiva en cinco pacientes (casos 371, 511, 267, 958-3 y 963). Es decir que las pruebas específicas para cada virus permitieron confirmar la infección por arbovirus en todos los pacientes evaluados: dos presentaron infección por el DENV, dos por el CHIKV y cuatro presentaron infección simultánea con los dos virus.

La infección con el DENV se confirmó en un paciente de diez años de edad mediante RT-PCR (caso 420). Este menor presentó signos asociados con dengue grave, como alteración cardiopulmonar, derrame pleural, hepatomegalia, trombocitopenia y dolor abdominal, por lo cual requirió cuidados intensivos y hospitalización prolongada. El segundo caso correspondió a un neonato de 16 días de edad con IgM positiva (caso 958-3). Con base en el cuadro clínico y los resultados de las pruebas de laboratorio, se concluyó que se trataba de un caso de dengue sin signos de alarma, aunque el diagnóstico presuntivo registrado fue de sepsis neonatal. En este caso no se detectó el CHIKV, pero no se descarta una posible infección simultánea, dado que sus padres y hermanas reportaron que habían presentado los síntomas recientemente.

En el caso 986-1, un paciente de cuatro días de nacido, se detectó únicamente infección por el CHIKV, con signos graves como alteraciones neurológicas y trombocitopenia. En el momento del ingreso, este paciente fue diagnosticado con ictericia fisiológica y sospecha de infección por el CHIKV. No fue posible confirmar que se tratara de transmisión vertical, dado que no se contaba con una muestra de la madre para las pruebas respectivas. El segundo caso correspondió a un niño de cinco meses de edad (caso 519), quien presentó alteraciones neurológicas graves y fue diagnosticado con síndrome convulsivo generalizado; además, se detectó Streptococcus pneumoniae en el líquido cefalorraquídeo, lo cual es indicativo de una meningitis bacteriana.

Se confirmaron infecciones simultáneas en cuatro pacientes: dos neonatos, un niño de dos meses y uno de cuatro años. En los casos de los neonatos de siete y cinco días de edad, el diagnóstico presuntivo en el momento del ingreso hospitalario fue de sepsis bacteriana (casos 371 y 511) y, 
Cuadro 2. Diagnóstico presuntivo y diagnóstico confirmado. La información contenida en las historias clínicas se clasificó como hallazgos clínicos, hallazgos de laboratorio, observaciones generales y diagnóstico presuntivo. Los resultados de las pruebas serológicas y virológicas se presentan como lgM para dengue y PCR con transcriptasa inversa. Para el diagnóstico confirmado, se tuvieron en cuenta los resultados de las dos pruebas.

\begin{tabular}{|c|c|c|c|c|c|c|c|}
\hline Código & Edad & Hallazgos clínicos & Hallazgos paraclínicos & $\begin{array}{l}\text { Diagnóstico } \\
\text { presuntivo }\end{array}$ & $\begin{array}{c}\text { lgM } \\
\text { dengue }\end{array}$ & $\begin{array}{c}\text { PCR con } \\
\text { transcriptasa inversa }\end{array}$ & $\begin{array}{l}\text { Diagnóstico } \\
\text { confirmado }\end{array}$ \\
\hline 371 & $\begin{array}{c}7 \\
\text { días }\end{array}$ & $\begin{array}{l}\text { Erupción cutánea } \\
\text { Debilidad } \\
\text { Hipoactividad }\end{array}$ & Hemocultivo: negativo & $\begin{array}{l}\text { Sepsis bacteriana } \\
\text { Dengue o } \\
\text { chikungunya }\end{array}$ & Positiva & $\begin{array}{l}\text { CHIKV: positiva } \\
\text { DENV: negativa }\end{array}$ & $\begin{array}{l}\text { Infección } \\
\text { simultánea }\end{array}$ \\
\hline 11 & $\begin{array}{c}5 \\
\text { días }\end{array}$ & $\begin{array}{l}\text { Erupción cutánea } \\
\text { Dificultad respiratoria } \\
\text { Hipoactividad } \\
\text { Debilidad } \\
\text { Disminución del apetito }\end{array}$ & $\begin{array}{l}\text { Radiografía de tórax: } \\
\text { aumento en densidad } \\
\text { de hilios pulmonares } \\
\text { Cambios inflamatorios bronquíticos } \\
\text { Sin derrame pleural } \\
\text { Sin evidencia de foco } \\
\text { bronconeumónico }\end{array}$ & $\begin{array}{c}\text { Sepsis neonatal } \\
\text { tardía }\end{array}$ & Positiva & $\begin{array}{l}\text { CHIKV: positiva } \\
\text { DENV: positiva para } \\
\text { serotipo } 1\end{array}$ & $\begin{array}{l}\text { Infección } \\
\text { simultánea }\end{array}$ \\
\hline 267 & $\begin{array}{c}2 \\
\text { meses }\end{array}$ & $\begin{array}{l}\text { Dificultad respiratoria: } \\
\text { episodio de apnea y cianosis } \\
\text { Alteración neurológica } \\
\text { Exantema macular } \\
\text { generalizado } \\
\text { Astenia y adinamia } \\
\text { Debilidad } \\
\text { Disminución del apetito }\end{array}$ & $\begin{array}{l}\text { Radiografía de tórax: } \\
\text { foco bronconeumónico } \\
\text { parahiliar bilateral } \\
\text { Sin derrame pleural } \\
\text { Silueta cardíaca de } \\
\text { tamaño normal } \\
\text { Transfusión de glóbulos } \\
\text { rojos empacados }\end{array}$ & $\begin{array}{l}\text { Broncoaspiración } \\
\text { Paro cardiaco } \\
\text { revertido } \\
\text { Dengue o } \\
\text { chikungunya }\end{array}$ & Positiva & $\begin{array}{l}\text { CHIKV: positiva } \\
\text { DENV: negativa }\end{array}$ & $\begin{array}{l}\text { Infección } \\
\text { simultánea }\end{array}$ \\
\hline 519 & $\begin{array}{c}5 \\
\text { meses }\end{array}$ & $\begin{array}{l}\text { Alteración neurológica: } \\
\text { somnolencia } \\
\text { Convulsiones generalizadas } \\
\text { Astenia y adinamia } \\
\text { Debilidad } \\
\text { Disminución del apetito }\end{array}$ & $\begin{array}{l}\text { TC cerebral sin evidencia } \\
\text { de lesión intraaxial o extraaxial } \\
\text { Radiografía de tórax: infiltrado en } \\
\text { lóbulo medio con consolidación } \\
\text { Punción lumbar: } \\
\text { cocos Gram positivos en líquido } \\
\text { cefalorraquídeo } \\
\text { Reacción leucocitaria escasa } \\
\text { S. pneumoniae en cultivo } \\
\text { en líquido cefalorraquídeo }\end{array}$ & $\begin{array}{c}\text { Síndrome } \\
\text { convulsivo } \\
\text { generalizado }\end{array}$ & Negativa & $\begin{array}{l}\text { CHIKV: positiva } \\
\text { DENV: negativo }\end{array}$ & Chikungunya \\
\hline $958-3$ & $\begin{array}{c}16 \\
\text { días }\end{array}$ & $\begin{array}{l}\text { Dolor al moverse } \\
\text { Exantema macular en } \\
\text { abdomen y miembro inferior } \\
\text { Alteración neurológica: } \\
\text { irritabilidad } \\
\text { Hipoactividad } \\
\text { Debilidad } \\
\text { Disminución del apetito }\end{array}$ & $\begin{array}{l}\text { Hemograma: } \\
\text { Hemoglobina: } 12.9 \mathrm{~g} / \mathrm{dl} \\
\text { Hematocrito: } 37,0 \% \\
\text { Leucocitos: } 8.760 / \mathrm{mm}^{3} \\
\text { Plaquetas: } 413.000 / \mathrm{mm}^{3}\end{array}$ & $\begin{array}{l}\text { Sepsis neonatal } \\
\text { Chikungunya }\end{array}$ & Positivo & $\begin{array}{l}\text { CHIKV: negativa } \\
\text { DENV: negativa }\end{array}$ & $\begin{array}{l}\text { Dengue } \\
\text { (Probable } \\
\text { CHIKV) }\end{array}$ \\
\hline 963 & $\begin{array}{c}4 \\
\text { años }\end{array}$ & $\begin{array}{l}\text { Exantema en } \\
\text { extremidades } \\
\text { Debilidad } \\
\text { Astenia y adinamia }\end{array}$ & $\begin{array}{l}\text { Hemograma } \\
\text { Hemoglobina: } 14.1 \mathrm{~g} / \mathrm{dl} \\
\text { Hematocrito: } 41.7 \% \\
\text { Leucocitos: } 4.170 / \mathrm{mm}^{3} \\
\text { Plaquetas: } 176.000 / \mathrm{mm}^{3}\end{array}$ & NR & Positivo & $\begin{array}{l}\text { CHIKV: positiva } \\
\text { DENV: negativa }\end{array}$ & $\begin{array}{l}\text { Infección } \\
\text { simultánea }\end{array}$ \\
\hline $986-1$ & $\begin{array}{c}4 \\
\text { días }\end{array}$ & $\begin{array}{l}\text { Ictericia } \\
\text { Dolor al moverse } \\
\text { Alteración neurológica: } \\
\text { irritabilidad } \\
\text { Erupción cutánea } \\
\text { Hipoactividad }\end{array}$ & $\begin{array}{l}\text { Hemocultivo: negativo } \\
\text { a los cinco días } \\
\text { Hemoglobina: } 19.8 \mathrm{~g} / \mathrm{dl} \\
\text { Hematocrito: } 55,6 \% \\
\text { Leucocitos: } 9.230 / \mathrm{mm}^{3} \\
\text { Plaquetas: } 54.000 / \mathrm{mm}^{3} \\
\text { PCR } 15,0 \mathrm{mg} / \mathrm{dl} \\
\text { Reticulocitos: } 2,9 \% \\
\text { Bilirrubina total: } 14,90 \mathrm{mg} / \mathrm{dl} \\
\text { Bilirrubina directa: } 0,50 \mathrm{mg} / \mathrm{dl} \\
\text { Bilirrubina indirecta: } 14,40 \mathrm{mg} / \mathrm{dl}\end{array}$ & $\begin{array}{l}\text { Ictericia fisiológica } \\
\text { Chikungunya }\end{array}$ & Negativo & $\begin{array}{l}\text { CHIKV: positiva } \\
\text { DENV: negativa }\end{array}$ & Chikungunya \\
\hline 420 & $\begin{array}{c}10 \\
\text { años }\end{array}$ & $\begin{array}{l}\text { Dolor osteomuscular } \\
\text { Astenia y adinamia } \\
\text { Erupción cutánea } \\
\text { Alteración cardiopulmonar } \\
\text { Derrame pleural } \\
\text { Dolor en la palpación } \\
\text { abdominal localizado en } \\
\text { hipocondrio derecho } \\
\text { Hepatomegalia } \\
\text { Edema palpebral } \\
\text { Petequias }\end{array}$ & $\begin{array}{l}\text { Ecocardiograma: insuficiencia } \\
\text { aórtica moderada } \\
\text { Leve hipertensión pulmonar } \\
\text { Paciente con foco neumónico } \\
\text { Hemograma: } \\
\text { Hemoglobina: } 9,1 \mathrm{~g} / \mathrm{dl} \\
\text { Hematocrito: } 27,8 \% \\
\text { Leucocitos: } 9.100 / \mathrm{mm}^{3} \\
\text { Plaquetas: } 88.000 / \mathrm{mm}^{3} \\
\text { Tiempo de protrombina: } 11,3 \mathrm{~s} \\
\text { Tiempo parcial de tromboplastina: } \\
33,2 \mathrm{~s} \\
\text { Hemocultivos negativos }\end{array}$ & NR & Negativo & $\begin{array}{l}\text { CHIKV: negativa } \\
\text { Dengue: positiva para } \\
\text { serotipo } 2\end{array}$ & Dengue \\
\hline
\end{tabular}

NR: dato no registrado; TC: tomografía computarizada 
en uno de ellos (371), se sospechó la presencia de infección por el DENV o el CHIKV debido a los antecedentes familiares. El paciente de dos meses de edad (caso 267), ingresado por broncoaspiración y sospecha de infección por el DENV o el CHIKV, presentó episodios de apnea y requirió asistencia respiratoria mecánica invasiva en la unidad de cuidados intensivos. El paciente de cuatro años (caso 963) presentó síntomas típicos leves, como exantema, debilidad e 'hipoactividad'.

\section{Discusión}

Las pruebas específicas de laboratorio permitieron determinar el agente etiológico causante de la enfermedad en los ocho casos de síndrome febril evaluados. En dos de ellos se detectó el DENV, en otros dos, el CHIKV, y en cuatro, infección simultánea con los dos virus. Con base en los hallazgos clínicos y en los antecedentes familiares, dos pacientes fueron diagnosticados como casos probables de dengue, cuatro como casos probables de chikungunya y tres como sepsis neonatal.

Estos resultados evidencian el gran problema de salud que enfrenta la población ubicada en zonas endémicas para dengue con la introducción del CHIKV, así como las dificultades del sistema de salud para dar un diagnóstico correcto y oportuno basado únicamente en la información clínica. Además, se pone de presente la vulnerabilidad de la población pediátrica frente a la infección con los dos arbovirus y a sus implicaciones a largo plazo, así como el subregistro de casos y las infecciones simultáneas.

En este estudio se confirmó la infección por el DENV en seis pacientes; uno de ellos se presentó como dengue grave, tres no presentaron signos de alarma y dos registraron dificultad respiratoria, por lo que se clasificaron como dengue con signos de alarma. Sin embargo, en los casos de infección simultánea con el CHIKV, estas complicaciones no pueden atribuirse con certeza a la infección por el DENV, pues también se han registrado como manifestaciones atípicas de la infección por el CHIKV $(17,18)$. Inicialmente, solo en dos casos se hizo un diagnóstico presuntivo de dengue y el caso de dengue grave no fue reconocido como tal, probablemente, porque no se detectaron anticuerpos IgM para el virus. El diagnóstico correcto de dengue es muy complejo y se dificulta más en áreas donde hay circulación del CHIKV, por lo cual se requieren pruebas específicas de laboratorio que, no solo confirmen la infección con el virus, sino que permitan detectar tempranamente los casos potencialmente graves. Una rápida confirmación de los casos durante la fase febril es crucial para el manejo adecuado del paciente y para prevenir la propagación de la enfermedad.

Tradicionalmente, la infección por el CHIKV se catalogaba como "benigna" y rara vez resultaba mortal, pero hoy es un problema de salud pública por el dolor incapacitante que produce, las secuelas a largo plazo que se han descrito (artritis, alteraciones neurológicas y sensoriales) y la diversidad de órganos que afecta (17-20). En este estudio se detectó el virus en tres neonatos y tres pacientes pediátricos, algunos de los cuales presentaron complicaciones neurológicas y respiratorias leves, como dificultad para respirar e irritabilidad, y graves, como apnea y síndrome convulsivo, las cuales podrían estar relacionadas con la infección viral $(17,21,22)$. Durante la gran epidemia en la isla de La Réunion entre marzo de 2005 y abril de 2006, se puso en evidencia la asociación entre la infección viral y las complicaciones neurológicas, cardiovasculares, respiratorias, hepáticas y dérmicas, entre otras. Las alteraciones neurológicas se registraron como la principal causa de muerte en individuos con infección grave, y se señaló que los recién nacidos y los ancianos habían sido los más propensos a presentar estas complicaciones $(21,22)$.

El diagnóstico preciso de las infecciones por el CHIKV y el DENV en neonatos, es un desafío imposible de resolver sin pruebas específicas para cada virus. Hay varios reportes de infecciones por dengue y chikungunya en neonatos asociadas con la transmisión vertical, las cuales con frecuencia se confunden con sepsis bacteriana, lo que lleva a la administración innecesaria de antibióticos hasta que el diagnóstico viral se confirma mediante pruebas serológicas o moleculares (23-26). Es posible confirmar la transmisión materna del virus cuando los síntomas en el neonato aparecen antes de cumplir la primera semana y la madre presenta un caso confirmado de infección durante la última semana de embarazo. Este no fue el caso en el presente estudio, ya que no se contaba con las muestras de ninguna de las madres. La transmisión vertical se ha descrito en zonas tropicales donde el dengue es endémico, como el sudeste asiático, India, Brasil, Colombia, Centroamérica y el Caribe (23-26). Los síntomas en los neonatos aparecen hacia el cuarto día de nacidos, y se acompañan principalmente de trombocitopenia y exantema 
petequial; aunque la mayoría de los niños se recupera rápidamente, también se han reportado casos de dengue grave e, incluso, de muerte (23-26).

La fiebre del chikungunya neonatal se ha descrito en varios brotes epidémicos en Asia, África, la isla de La Réunion y, recientemente, en el continente americano (27-29). En una serie de ocho casos en Colombia, se observó una alta tasa de letalidad (38\%) y manifestaciones clínicas graves, como complicaciones cardiorrespiratorias, enterocolitis necrosante, miocarditis y meningoencefalitis (27). Las características clínicas frecuentes en los recién nacidos durante la epidemia en India, fueron fiebre, erupción cutánea, episodios de apnea inexplicables e hiperpigmentación difusa (29), mientras que en la isla La Réunion, en 10 de 19 casos de transmisión vertical se produjeron cuadros graves (nueve de edema cerebral y dos de hemorragia cerebral) y cuatro desarrollaron discapacidad persistente (deficiencia ocular y conductual o postural) (30).

El seguimiento de 33 niños infectados al nacer mostró que, a los dos años de edad, $51 \%$ de ellos tenía un retraso en el desarrollo neurológico global en comparación con los no infectados; las habilidades de coordinación y del lenguaje se vieron afectadas con mayor frecuencia que las de movimiento, postura y sociabilidad (31). Esos resultados sugieren que la infección por el CHIKV adquirida en el período perinatal puede ser grave y generar una discapacidad permanente. Por lo tanto, se sugiere hacer el seguimiento de los neonatos infectados con el CHIKV para descartar la aparición de secuelas a largo plazo.

En este estudio se reportan cuatro casos de infección simultánea en pacientes pediátricos, dos de ellos neonatos, lo cual sugiere que hay transmisión vertical de los dos virus. Las infecciones simultáneas por el DENV y el CHIKV no son infrecuentes y se han descrito en zonas donde el dengue es endémico, como India, el sudeste de Asia, África y la isla de San Martín en América. El porcentaje de infección con los dos virus varía entre $3 \%$ (Gabón y San Martín) y $18,2 \%$ en Madagascar durante el brote epidémico del 2006 (32-34); sin embargo, existen pocos registros en pacientes pediátricos y ninguno en neonatos. Durante el brote epidémico del CHIKV de 2008-2009 en Tailandia, se analizó una cohorte de 50 niños menores de 15 años de edad, 32 de los cuales fueron positivos para el CHIKV y diez para el DENV, y solo se detectó una infección simultánea con los dos virus (35), lo cual indicaría que para este grupo etario la tasa de infección concomitante es baja. No obstante, en otro estudio con cerca de 550 pacientes en la zona este de la India, se encontraron 131 muestras positivas para el CHIKV, 104 para el DENV y 68 infecciones simultáneas, seis de ellas en menores de 15 años, es decir, un porcentaje de 8,8 (36).

En este estudio se documenta la importancia y la urgencia de implementar métodos de diagnóstico de mayor especificidad y sensibilidad que puedan distinguir una infección por el CHIKV de una causada por el DENV, ya que el primero puede producir secuelas neurológicas de consideración y el DENV causa una enfermedad hemorrágica grave. Un diagnóstico adecuado es importante, no solo para el manejo correcto del paciente, el control de la transmisión y la vigilancia epidemiológica, sino también, para obtener información más completa sobre las manifestaciones clínicas de las dos infecciones.

\section{Agradecimientos}

Al Centro de Investigación del Hospital Universitario de La Samaritana, Bogotá, y al Hospital Universitario de La Samaritana-Unidad Funcional Girardot.

\section{Conflicto de intereses}

Manifestamos no tener conflictos de intereses con respecto al artículo.

\section{Financiación}

Proyecto financiado por el Programa de la Red de Investigación Multidisciplinaria para la prevención y control de enfermedades transmitidas por vectores, Colciencias, 360-2011.

\section{Referencias}

1. Bolling B, Weaver $\mathbf{S}$, Tesh $\mathbf{R}$, Vasilakis $\mathbf{N}$. Insect-specific virus discovery: Significance for the arbovirus community. Viruses. 2015;7:4911-28. http://dx.doi.org/10.3390/v7092851

2. Rezza G. Dengue and chikungunya: Long-distance spread and outbreaks in naïve areas. Pathog Glob Health. 2014;108: 349-55. http://dx.doi.org/10.1179/2047773214Y.0000000163

3. Instituto Nacional de Salud. Boletín Epidemiológico Semanal. Sivigila. Semana 38 de 2015. Fecha de consulta: 3 de octubre de 2015. Disponible en: http://www.ins.gov.co/ boletin-epidemiologico/Boletn\%20Epidemiolgico/2015\%20 Boletin\%20epidemiologico\%20semana\%2038.pdf

4. Hoz JM, Bayona B, Viloria S, Accini JL, Juan-Vergara HS. Fatal cases of Chikungunya virus infection in Colombia: Diagnostic and treatment challenges. J Clin Virol. 2015;69: 27-9. http://dx.doi.org/10.1016/j.jcv.2015.05.021

5. Mondragón-Cardona AE, Villamil-Gómez WE, RodríguezMorales A. Chikungunya congénito y neonatal: problemas emergentes en pediatría en Latinoamérica. Pediatr (Asunción). 2015;42:149-51. http://dx.doi.org/10.18004/ped. 2015.agosto.149-151 
6. Cardona-Ospina JA, Henao-San Martín V, PanizMondolfi AE, Rodríguez-MA. Mortality and fatality due to Chikungunya virus infection in Colombia. J Clin Virol. 2015;70:14-5. http://dx.doi.org/10.1016/j.jcv.2015.07.001

7. Restrepo AC, Baker P, Clements CA. National spatial and temporal patterns of notified dengue cases, Colombia 20072010. Trop Med Int Heal. 2014;19:863-71. http://dx.doi. org/10.1111/tmi. 12325

8. Caglioti C, Lalle E, Castilletti C, Carletti F, Capobianchi MR, Bordi L. Chikungunya virus infection: An overview. New Microbiol. 2013;36:211-27.

9. Horstick O, Jaenisch T, Martínez E, Kroeger A, See LLC, Farrar J, et al. Comparing the usefulness of the 1997 and 2009 WHO dengue case classification: A systematic literature review. Am J Trop Med Hyg. 2014;91:621-34 http://dx.doi.org/10.4269/ajtmh.13-0676

10. San Martín JL, Brathwaite $\mathbf{O}$, Zambrano B, Solórzano JO, Bouckenooghe A, Dayan GH, et al. The epidemiology of dengue in the Americas over the last three decades: $A$ worrisome reality. Am J Trop Med Hyg. 2010;82:128-35. http://dx.doi.org/10.4269/ajtmh.2010.09-0346

11. Castrillón JC, Carlos J, Urcuqui S. Dengue en Colombia: diez años de evolución. Rev Chil Infectol. 2015;32:142-9. http://dx.doi.org/10.4067/S0716-10182015000300002

12. Instituto Nacional de Salud. Boletín Epidemiológico Semanal. Sivigila. Semana 53 de 2014. Fecha de consulta: 3 de octubre de 2015. Disponible en: http://www.ins.gov.co/ boletin-epidemiologico/Boletn\%20Epidemiolgico/2014\%20 Boletin\%20epidemiologico\%20semana\%2053.pdf

13. Organización Panamericana de la Salud. Número de casos de fiebre Chikungunya en las Américas, 2015. Fecha de consulta: 3 de octubre 3 de 2015. Disponbile en: http:// www.paho.org/hq/index. php? Itemid=40931

14. Castellanos JE, Coronel-Ruíz C. Dengue disease diagnosis: A puzzle to be solved. Rev Fac Med. 2014;62:455-64. http://dx.doi.org/10.15446/revfacmed.v62n4.45593

15. Chien L, Liao T, Shu P, Gubler DJ, Chang GJ, Huang $J$. Development of real-time reverse transcriptase PCR assays to detect and serotype dengue viruses. J Clin Microbiol. 2006;44:1295-304. http://dx.doi.org/10.1128/JCM. 44.4.1295-1304.2006

16. Pastorino B, Bessaud M, Grandadam M, Murri S, Tolou HJ, Peyrefitte CN. Development of a TaqMan RTPCR assay without RNA extraction step for the detection and quantification of African Chikungunya viruses. J Virol Methods. 2005;124:65-71. http://dx.doi.org/10.1016/j. jviromet.2004.11.002

17. Renault P, Solet JL, Sissoko D, Balleydier E, Larrieu $\mathrm{S}$, Filleul $\mathrm{L}$, et al. A major epidemic of chikungunya virus infection on Reunion Island, France, 2005-2006. Am J Trop Med Hyg. 2007;77:727-31.

18. Her Z, Kam YW, Lin RTP, Ng LF. Chikungunya: A bending reality. Microbes Infect. 2009;11:1165-76. http://dx.doi. org/10.1016/j.micinf.2009.09.004

19. Gérardin P, Fianu A, Malvy D, Mussard C, Boussaïd K, Rollot $\mathrm{O}$, et al. Perceived morbidity and community burden after a Chikungunya outbreak: The TELECHIK survey, a population-based cohort study. BMC Med. 2011;9:5. http:// dx.doi.org/10.1186/1741-7015-9-5
20. Chopra A, Anuradha V, Lagoo-Joshi V, Kunjir V, Salvi S, Saluja M. Chikungunya virus aches and pains: An emerging challenge. Arthritis Rheum. 2008;58:2921-2. http//dx.doi. org/10.1002/art.23753

21. Economopoulou A, Domínguez M, Helynck B, Sissoko D, Wichmann O, Quenel P, et al. Atypical Chikungunya virus infections: Clinical manifestations, mortality and risk factors for severe disease during the 2005-2006 outbreak on Reunion. Epidemiol Infect. 2009;137:534-41. http//dx. doi.org/10.1017/S0950268808001167

22. Tandale BV, Sathe PS, Arankalle VA, Wadia RS, Kulkarni R, Shah SV, et al. Systemic involvements and fatalities during Chikungunya epidemic in India, 2006. J Clin Virol. 2009;46:145-9. http://dx.doi.org/10.1016/j.jcv. 2009.06.027

23. Choudhry SP, Gupta RK, Kishan J. Dengue shock syndrome in new born - A case series. Indian Pediatr. 2004;41:397-9.

24. Sirinavin S, Nuntnarumit $\mathbf{P}$, Supapannachart $\mathbf{S}$, Boonkasidecha S, Techasaensiri C, Yoksarn S. Vertical dengue infection: Case reports and review. Pediatr Infect Dis J. 2004;23:1042-7. http://dx.doi.org/10.1097/01.inf. $0000143644.95692 .0 \mathrm{e}$

25. Maroun SL, Marliere RC, Barcellus RC, Barbosa CN, Ramos JR, Moreira M. Case report: Vertical dengue infection. J Pediatr (Rio J). 2008;84:556-9. http://dx.doi. org/10.1590/S0021-75572008000700014

26. Salgado DM, Rodríguez JA, Lozano LD, Zabaleta TE. Perinatal dengue. Biomédica. 2013;33(Suppl.1):14-21. http://dx.doi.org/10.7705/biomedica.v33i0.1449

27. Villamil-Gómez W, Alba-Silvera L, Menco-Ramos A, González-Vergara A, Molinares-Palacios T, BarriosCorrales $\mathrm{M}$, et al. Congenital chikungunya virus infection in Sincelejo, Colombia: A case series. J Trop Pediatr. 2015;61:386-92. http://dx.doi.org/10.1093/tropej/fmv051

28. Rolón P, Fonseca R, Genes L, Pereira S, Zapatta L. Chikungunya adquirida en recién nacidos. Reporte de caso. Pediatr (Asunción). 2015;42:42-7. http://dx.doi.org/10.18004/ ped.2015.abril.42-47

29. Passi GR, Khan YZ, Chitnis DS. Chikungunya infection in neonates. Indian Pediatr. 2008;45:240-1.

30. Gérardin P, Barau G, Michault A, Bintner M, Randrianaivo H, Choker G, et al. Multidisciplinary prospective study of mother-to-child chikungunya virus infections on the island of La Réunion. PLoS Med. 2008;5:e60. http://dx.doi.org/10. 1371/journal.pmed.0050060

31. Gérardin P, Sampériz S, Ramful D, Boumahni B, Bintner M, Alessandri J-L, et al. Neurocognitive outcome of children exposed to perinatal mother-to-child chikungunya virus infection: The CHIMERE Cohort Study on Reunion Island. PLoS Negl Trop Dis. 2014;8:e2996. http://dx.doi. org/110.1371/journal.pntd.0002996

32. Leroy EM, Nkoghe D, Ollomo B, Nze-Nkogue C, Becquart P, Grard G, et al. Concurrent chikungunya and dengue virus infections during simultaneous outbreaks, Gabon, 2007. Emerg Infect Dis. 2009;15:591-3. http://dx.doi.org/10. 3201/eid1504.080664 
33. Omarjee R, Prat CM, Flusin O, Boucau S, Tenebray B, Merle $\mathbf{O}$, et al. Importance of case definition to monitor ongoing outbreak of chikungunya virus on a background of actively circulating dengue virus, St Martin, December 2013 to January 2014. Euro Surveil. 2014;19:1-3.

34. Ratsitorahina M, Harisoa J, Ratovonjato J, Biacabe $\mathrm{S}$, Reynes JM, Zeller $\mathrm{H}$, et al. Outbreak of dengue and chikungunya fevers, Toamasina, Madagascar, 2006. Emerg Infect Dis. 2008;14:1135-7. http://dx.doi.org/10.3201/eid1407. 071521
35. Laoprasopwattana K, Suntharasaj T, Petmanee $\mathbf{P}$, Suddeaugrai O, Geater A. Chikungunya and dengue virus infections during pregnancy: Seroprevalence, seroincidence and maternal-fetal transmission, southern Thailand, 20092010. Epidemiol Infect. 2015;1-8. http://dx.doi.org/10.1017/ S0950268815001065

36. Taraphdar D, Sarkar A, Mukhopadhyay BB, Chatterjee S. A comparative study of clinical features between monotypic and dual infection cases with chikungunya virus and dengue virus in West Bengal, India. Am J Trop Med Hyg. 2012;86:720-3. http://dx.doi.org/10.4269/ajtmh.2012.11-0704 